\title{
Don Quijote como figura identificadora y metáfora política en la Latinoamérica del siglo XIX
}

\author{
Strosetzki, Christoph
}

First published in:

Schmidt-Welle, Friedhelm; Simson, Ingrid (Hrsg.): El Quijote en América. Amsterdam : Rodopi, 2010, S. 73-83

ISBN: 978-90-420-3051-0

(C) 2010 Editions Rodopi B.V., Amsterdam 


\title{
Don Quijote como figura identificadora y metáfora política en la Latinoamérica del siglo XIX
}

\author{
Christoph Strosetzki
}

Resumen: Autores latinoamericanos del siglo XIX y XX como José Joaquín Fernández de Lizardi con su Periquillo Sarniento, Juan Montalvo con sus Capitulos que se le olvidaron a Cervantes, Juan Bautista Alberdi con su Peregrinación de Luz del Día o Viaje y aventuras de la Verdad en el Nuevo Mundo, Tulio Febres Cordero con Don Quijote en América o sea la cuarta salida del ingenioso hidalgo de la Mancha, José Rodó con su ensayo Ariel, al igual que Rubén Darío, José Vasconcelos, Leopoldo Zea y Octavio Paz entregan al personaje Don Quijote una dimensión política que vincula la novela cervantina a la conciencia de identidad hispanoamericana.

La gloriosa existencia de Don Quijote tiene una explicación muy peculiar que no se le tiene que agradecer a Cervantes, dice Unamuno, sino a sus numerosos lectores. Ser quijotista, y no cervantista, se convirtió precisamente en Latinoamérica en una de las divisas más populares. A la identificación potencial de un quijotista con Don Quijote, se le añade el hecho de que la cuestión de la identidad es central en la novela de Cervantes, lo que viene a enriquecer nuestro tema con una segunda dimensión.

Numerosos escritores latinoamericanos se plantearon por un igual cuestiones sobre la identidad. Estos escritores querían caracterizar no sólo la autonomía literaria, mejor dicho, el vínculo con modelos literarios, sino también la identidad específica del propio país o continente. A continuación se mostrará que perseguían tales deseos, principalmente en el siglo XIX y XX, refiriéndose a la novela o a la figura de Don Quijote.

El Periquillo Sarniento es considerado como la primera novela colonial latinoamericana. Tras su publicación en 1816, su autor, el mexicano Fernández de Lizardi (1776-1827), se ve expuesto a los ataques de los críticos. Se justifica en una "Apología" y rebate el reproche de carecer de realismo comparando su novela con el Quijote. 
Su propia interpretación realista no se diferenciaría mucho de la de Cervantes:

[...] yo tomo el Quijote de Cervantes y no veo en su acción nada extraordinario, nada prodigioso. Todos los sucesos son demasiado vulgares y comunes, tales como pudieran acontecer a un loco de las circunstancias de don Alonso Quijada. ${ }^{1}$

Asimismo, las numerosas inserciones moralizadoras las justifica de igual modo refiriéndose a Don Quijote: Don Quijote también moralizaba y predicaba a cada paso, y tanto que su criado le decía que podía coger un púlpito en las manos y andar por esos mundos predicando lindezas (Fernández de Lizardi 1980: 15).

El problema de la lectura, motivo fundamental en el Quijote, también fue de vital importancia para Lizardi. En su novela La Quijotita y su prima (1818), que ya alude a Don Quijote en el título, la protagonista -como la posterior Mme Bovary de Flaubert-representa un ejemplo del daño que provoca la lectura excesiva. ${ }^{2}$ Es curioso, por tanto, que el autor -el primero que a comienzos del siglo XIX escribe novelas en una Latinoamérica recién independizada de España- no sólo en su justificación del realismo, sino también en el uso de las técnicas narrativas y en la elección de la temática, se base conscientemente y en numerosas ocasiones en el Quijote, y así lo exprese explícitamente.

Juan Montalvo (1832-1889) no se queda atrás cuando en la segunda mitad del siglo XIX publica una imitación del original bajo el título de Capítulos que se le olvidaron a Cervantes. En Montalvo, como en Lizardi, Don Quijote es el modelo indiscutible. Los Capitulos de Montalvo se pueden calificar verdaderamente de pastiche del original, manteniéndose el estilo, la constelación de las figuras y el colorido local (véase Montalvo 1972). Que también existían otras posibilidades, lo muestra la obra de Juan Bautista Alberdi, Peregrinación de Luz del Día o Viaje y aventuras de la Verdad en el Nuevo Mundo del año 1875, en la que se aplican elementos de la obra de Cervantes a la situación latinoamericana (véase Alberdi 1916). A causa de la presupuesta familiarización con el Quijote se origina de ello un cierto efecto enajenante.

La narración de Alberdi se basa en los diálogos de Luciano y de Fontenelle. Se la puede comparar con las Lettres persanes de Montes- 
quieu o con Candide de Voltaire, en tanto que aporta el marco para numerosas consideraciones políticas y moral-filosóficas. "Verdad", es decir, "Luz del día" viaja por el Nuevo Mundo y se encuentra con figuras literarias como Tartufo, Gil Blas o Basilio, ahora convertidos en bribones. Finalmente, ella se lanza a la búsqueda de los representantes de la vieja caballería española: El Cid, Pelayo y Don Quijote.

Mientras que éste último, que a causa de la lectura de novelas de caballería confunde los molinos de viento con gigantes, domina ahora en América sobre los ciudadanos, tan sólo en virtud de sus propios decretos, promulgados por escrito, confiando ciegamente en su realización, un hecho que aclara al lector la discrepancia entre el texto constitucional democrático y la realidad política en esta época. Mientras que, por un lado, el Don Quijote de Cervantes se deja inducir, por medio de la lectura de novelas de caballería, a crearse una falsa interpretación de la realidad, este Quijote americano, por otro lado, se vuelve loco con sus propios textos. Éstos, a su vez, resultan de la lectura de libros sobre los más recientes proyectos social-políticos y teorías políticas.

Asimismo, comete un segundo error muy grave en el diseño de su estado, al que llama "Quijotanía”: su electorado está formado básicamente por carneros. La duda de su escudero sobre la madurez política de este pueblo la disipa refiriéndose a Darwin. De acuerdo con éste prueba que con una adecuada aceleración del proceso de evolución y mediante una buena educación, los carneros, en un par de años y sin dificultades, serían capaces de llevar a cabo el mismo desarrollo que el que habían experimentado los monos antes que ellos. Cuando su criado duda porque teme la burla de los demás, Don Quijote disipa rápidamente sus temores:

Toda la diferencia que separa el pueblo de "Quijotanía" de los otros pueblos cuya risa temes, es que los habitantes del nuestro son ciudadanos en forma de carneros, mientras los otros son carneros en forma de ciudadanos. (Alberdi 1916: 170).

Puesto que la votación en los plebiscitos se da con la palabra "mäh", el criado percibe que esto en francés significa 'mais', es decir, se trata de una negación. No obstante, Don Quijote le asegura que se trata de una forma modificada de la palabra inglesa "yeah", es decir, 'yes'. 
Al contrario que en Lizardi y en Montalvo, en Alberdi no predomina el intento de orientarse en el Quijote de Cervantes. Alberdi, sin embargo, parece que es diferente, ya que sus figuras y constelación de figuras tienen otras funciones situadas en un contexto nuevo. Ya no es el mundo de las novelas de caballería el motivo de la parodia, sino la fe ciega en la realización de las reformas democráticas a través de decretos basados en teorías políticas modernas. Alberdi parodia una inapropiada visión de futuro reinante en el presente, mientras que Cervantes critica una inadecuada orientación en el mundo de los caballeros andantes de antaño.

Ya en el prólogo del Don Quijote en América o sea la cuarta salida del ingenioso hidalgo de la Mancha de Tulio Febres Cordero, publicado por primera vez en 1905, se pone en evidencia que su intención difiere de la de Lizardi y de la de Montalvo. Él quería escribir una obra en la que la historia, la tradición y los mitos de Latinoamérica, especialmente de Venezuela, ocuparan una posición central. Quería utilizar también el modelo de Cervantes, pero referirse a su presente. En eso no perseguía primeramente fines literarios, sino más bien patrióticos. Señaló que con su narración pretendía oponerse a las ideas de progreso, al desprecio hacia los criollos y a la esclava imitación de todo lo extranjero. Se trata pues de un libro entretenido con una clara referencia a las ideas del momento.

Es por ello que Don Quijote tiene que aclarar al principio a Sancho que ahora viven en otro país y en otra época; tienen que acostumbrarse a un país en el que los conceptos de honor y galantería han sido sustituidos por los de progreso y libertad. Es por esta razón que no quiere perder el tiempo pensando en una Dulcinea: "la dama de mis pensamientos, la reina y señora de mi voluntad es únicamente la gran idea, la idea santa y esplendorosa del progreso moderno". (Febres Cordero 1960: 36). Sancho le asiste como secretario, como ayudante en un laboratorio químico, como editor de sus libros y, finalmente, como acompañante. El mismo Don Quijote se pone un nuevo nombre y se plantea una nueva tarea: "Yo soy el doctor Alonso Quix, caballero de la orden del Progreso, ciudadano cosmopolita, instructor y mecenas del pueblo, y reformador de viejas costumbres". (Febres Cordero 1960: 47).

Quix no sólo cree en el progreso, sino que también lo fomenta desarrollando él mismo un medicamento (cap. 8) o descubriendo un heliógrafo, que absorbe rayos de sol durante el día y brilla por la no- 
che. La búsqueda de la sustancia que necesita para ello le hace viajar por las selvas tropicales americanas. Las experiencias que allí vive se alternan con numerosos episodios y narraciones intercaladas. Al llegar a un pueblo latinoamericano saluda alegremente al pueblo soberano de América, el cual se había liberado del yugo de una servidumbre centenaria y del despotismo psicológico de unas tradiciones anticuadas para acoger, ahora más que nunca, los ideales de la razón, la libertad y el progreso en todos los sentidos (véase Febres Cordero 1960: 120). En Mapiche ven en él la personificación de estas ideas, ven a un "hombre superior, cosmopolita y habituado a la vida moderna en los grandes centros". (Febres Cordero 1960: 130).

Significativamente, el hotel "Posada del fraile" se pasa a llamar "Hotel Cosmopolita" en su honor. ${ }^{3}$ El final de la obra parece ser también una parodia clara sobre el progreso cuando el Dr. Quix realiza un experimento con el globo cautivo y no consigue retenerlo. Se eleva a tales alturas que se pierde en la lejanía y desde entonces nadie lo vuelve a ver.

Aquí no se parodia, pues, un mundo de valores tradicionales y obsoletos, como Cervantes vio en los libros de caballería, sino que el objeto de la parodia es más bien la idea de progreso que se propagó en numerosos escritos del siglo XIX. Como personificación del progreso se encuentra Dr. Quix en claro contraste con los demás habitantes de Latinoamérica, que le admiran tanto más cuanto menos le entienden. La adaptación de Febres Cordero de los nombres, los temas y la constelación de figuras al contexto latinoamericano de finales del siglo XIX se basa en primer lugar en la idea sobre el progreso, introducida del extranjero, que desvirtúa el sentido de la propia realidad.

Mientras que en los ejemplos precedentes, la imitación literaria ocupaba el primer plano de la obra, en éste debe centrarse la atención más bien en el ideal que se personifica en la figura de Don Quijote. A este ideal se opone también el contrario, que personifica lo diferente, lo que se rechaza.

Este pensar polarizador empezó con el argentino Domingo Faustino Sarmiento (1811-1888), que diferencia entre civilización y barbarie. En la última ve el atraso que también quiere criticar en su novela Facundo, del año 1845, como lo criticó, según él, Cervantes en El Quijote. ${ }^{4}$ La barbarie es sinónimo de absolutismo, esclavitud, vandalismo y tradiciones obsoletas, mientras que la civilización se identifica con hegemonía constitucional, libertad y civilización europea. 
Sarmiento no ve encarnada la libertad en el autoritarismo de los gauchos, que viven en el campo, sino la barbarie, que encuentra su continuación en los caudillos. Esto explicaría las guerras civiles y los despotismos que tienen lugar tras la liberación de la hegemonía colonial. Por ello, Sarmiento tan sólo ve cierto grado de civilización en las ciudades con influencia europea.

José Rodó (1871-1917) encuentra un nuevo contenido para la oposición entre civilización y barbarie. Su ensayo Ariel, ${ }^{5}$ publicado en 1900 y tan frecuentemente citado, parte del drama filosófico de Renan, Caliban (1878), y de The Tempest de Shakespeare. Impresionado por la derrota española de 1898, identificó a los Estados Unidos con Calibán y a Latinoamérica con Ariel. Para Rodó, Calibán personificaba la barbarie anglosajona de signo utilitarista a la que confrontó con la civilización del área mediterránea, marcada por el cristianismo y la antigüedad griega y personificada en Ariel. Rodó invita a aferrarse a los valores españoles tradicionales y a no dejarse desviar por los esfuerzos panamericanos de Norteamérica que conducen hacia un pensamiento basado meramente en el utilitarismo y la negación de la propia identidad. El arielismo encontró gran resonancia entre los intelectuales latinoamericanos y motivó a Rubén Darío a dedicar su primer poema Cantos de vida y esperanza (1905) a Rodó.

Para Rodó, el gran logro de la época del renacimiento español es el descubrimiento y la conquista de América, que encuentran su máxima expresión en el Quijote (véase Uribe Echevarría 1949: 181). Mediante su crítica a los héroes de los antiguos libros de caballerías, Don Quijote habría dejado libre un lugar para un nuevo tipo de héroe, un lugar para los conquistadores Cortés y Pizarro. De este modo, la filosofía de Don Quijote sería al mismo tiempo la filosofía de los conquistadores del Nuevo Mundo, que aún sobrevive en el gaucho. Honrar al Quijote en Latinoamérica significa para Rodó también cuidar la relación con España, a la que se le debe agradecimiento como descubridora y portadora de la civilización (véase Rodó 1950: 1146-1148). La identificación entre Don Quijote y los conquistadores españoles hecha por Rodó la matizará con Asturias. Éste ve en Las Casas un Don Quijote que defendió a los indios y se levantó contra los conquistadores y colonizadores a pesar de todos los obstáculos. ${ }^{6}$

Al igual que Rodó, Rubén Darío (1867-1916) también ve en Don Quijote una figura de identificación. Para él, Don Quijote es un personaje verdaderamente mítico, por ello lo compara con Hércules. Como 
aquél, Hércules fue liberador de los oprimidos, protector de los débiles y sobresalto de los monstruos. Las salidas de Don Quijote se corresponden con los "trabajos" de Hércules. No obstante, éste sería el prototipo de la fuerza bruta, mientras que Don Quijote representa el ideal y el espíritu caballeresco. Puesto que Hércules no fue ningún jinete, a Darío le parece finalmente más acertado comparar a Don Quijote con Bellerofón, el jinete de Pegaso (véase Darío 1950: 555-559).

Esta interpretación heroizante se opone a la de su narración fantástica, influida por la derrota de las tropas españolas frente a las norteamericanas en Cuba y publicada bajo el título de "D.Q." en Buenos Aires en 1899 (véase Palau de Nemes 1981: 943-47). Darío utiliza este trasfondo histórico para representar el honor de la España vencida y la maldad del enemigo en la capitulación.

Cuando los españoles tuvieron que capitular y entregar sus armas, un oficial, cuyos bultos están marcados con las iniciales "D. Q.", se tira de un precipicio para salvar así su bandera. Finalmente, en el epílogo, un capellán está leyendo de un libro, del que no se cita ni el protagonista, ni el título, ni el autor. No obstante, queda claro que el oficial y Don Quijote son la misma persona: "Frisaba la edad de nuestro hidalgo con los cincuenta años. [...] Quieren decir que tenía el sobrenombre de Quijada o Quesada”. (Darío 1970: 145). Ante este trasfondo contemporáneo de Darío, el oficial Don Quijote representa la pérdida sentida y definitiva del poder español en Latinoamérica. Al mismo tiempo, "D. Q." aparece como precursor de los posteriormente publicados Cantos de vida y esperanza de Darío, que persiguen una y otra vez la defensa de los valores españoles. El poema más conocido a este respecto es el titulado "A Roosevelt", cuya persona está caracterizada como "futuro invasor de la América ingenua que tiene sangre indígena, que aún reza a Jesucristo y aún habla en español". (Darío 1965: 123). Darío contrapone a los fuertes y ricos Estados Unidos con la América de Colón y Moctezuma, marcada por los poetas. Como Rodó, al que aprecia mucho, identifica a Don Quijote con España, que también para él representa Latinoamérica. Eleva al héroe Don Quijote, en el que Rodó había visto una figura histórica muy significativa, al nivel de mito y de objeto de culto.

Al igual que Darío, para José Vasconcelos (México, 1882-1959) el Quijote está tan unido a la esencia y la lengua españolas que Latinoamérica puede considerar como propia la obra, así como España. Vasconcelos ve la historia latinoamericana estimulada por aquel "afán 
de universalidad, dirigido a lo maravilloso y lo eterno". (Vasconcelos 1947: 13), que también motivó el obrar de Don Quijote. Como ejemplos no sólo cita a Cortés y a Pizarro, sino que identifica a Don Quijote con todas las épocas y grandes hazañas de la historia latinoamericana:

[...] el Quijote que guió la conquista, el Quijote que después, durante la Colonia, expidió las leyes de Indias, el monumento jurídico más piadoso que vieron los siglos; el Quijote que más tarde hizo la independencia política, subsiste en nuestra historia [...]. (Vasconcelos 1947: 14).

La primacía latinoamericana la acentúa Vasconcelos, además, identificando las reglas de la caballería con las del cristianismo y viendo que la verdadera grandeza reside en el servicio del bien, en no darse por vencido aun cuando se pierda. Como ejemplo, Vasconcelos cita la imperturbabilidad quijotesca de Bolívar, que tenía la impresión de estar arando el mar y, sin embargo, no se le podía disuadir de su ideal. Así, para Vasconcelos Don Quijote personifica también una esperanza para el futuro de Latinoamérica.

Que la novela Don Quijote contiene una síntesis, que incluso se puede comprender también en la conceptualidad de la filosofía de la identidad, lo prueba el mexicano Leopoldo Zea (1912-2004). Este ve a Don Quijote como modelo para la superación de la contraposición entre idealismo y realismo. ${ }^{7} \mathrm{El}$ pensamiento racionalista y positivista, extendido a comienzos del siglo $\mathrm{XX}$, no le parece capaz de mediar entre la realidad de lo existente objetivamente y la idealidad de lo ambicionado subjetivamente. Una síntesis de este binomio opuesto es la que ve en la ingenuidad de Don Quijote. Sólo el ingenuo punto de vista de Don Quijote podría ganar de la realidad cotidiana una dimensión ideal, que Zea no teme en caracterizar con el concepto de lo "maravilloso". 8 La capacidad de sintetizar se habría perdido, por tanto, porque uno se habría acostumbrado a diferenciar entre el mundo real de la injusticia y el mundo ideal y ambicionado de la justicia.

Son dos líneas las que hacen a Zea llegar a su conclusión. Por un lado, parte del conocimiento fundamental de Ortega y Gasset apoyándose en José Gaos, discípulo de éste emigrado a México, de que en el Yo tiene lugar una síntesis entre el sujeto pensante y el objeto real: "Yo soy yo y mi circunstancia". Cuando Zea se sirve de esta tesis para definir su identidad mexicana tiene que partir, por otro lado, de la 
realidad de México. A comienzos del siglo XX México se encontraba bajo el gobierno de Porfirio Díaz, que creía que un fuerte póder estatal garantizaba un relativo bienestar a costa de los derechos de los ciudadanos. El grupo del "Ateneo de la Juventud"" se había dirigido contra el positivismo, fomentado por este gobierno, y a favor de la revalorización de intereses intelectuales; y son Vasconcelos y Zea quienes continúan sus pretensiones. Querían empezar con la educación del individuo para luego ponerle intelectualmente en la situación de hacer uso de sus derechos como ciudadanos en la realidad social. Con este trasfondo, se explica la definición del sujeto de Zea como síntesis entre idealidad y realidad. La situación histórica es, por tanto, la que motiva su interpretación de Don Quijote según la filosofía de la identidad, orientada en Ortega y Gasset. Se sabe que es el estudio del Quijote del que Ortega y Gasset considera haber extraído la clave de su sentencia "Yo soy yo y mi circunstancia". Así, el Quijote se encuentra tanto al comienzo como al final de esta discusión, que tiene que ver con la filosofía de la identidad.

Octavio Paz (1914-1998) acentúa, como Zea, la importancia de la subjetividad en el Quijote al proponer el siguiente título alternativo para la novela: "Las hazañas de la subjetividad o la conquista del mundo por la negación del mundo". (Paz 1988: 142). Así aclara la difícil relación entre realidad e idealidad, con la problemática sujetoobjeto de la teoría del conocimiento de fondo. Con ayuda de El Quijote, Paz explica la importancia de la subjetividad señalando la relación entre la perspectiva subjetiva y la duda de la realidad: dependiendo de si el lector hace caso a Sancho o a Don Quijote, tiene que ver con molinos de viento o con gigantes. Mientras que a Paz el mundo de Ariosto le parece absolutamente irreal, en el Quijote consta una interacción entre fantasía y realidad. A su vez, se observa una comunicación continuada entre necedad y sentido común. De manera diferente a Dante, en el que tanto el paraíso como el infierno se representan de un modo tan real como si se tratara de ciudades como Roma o Florencia, el árido paisaje de Castilla aparece en el Quijote como "un espejismo, un encantamiento de hechiceros. Hay una continuada oscilación entre lo real y lo irreal: los molinos son gigantes y un instante después son molinos". (Paz 1973: 23). La ambivalencia del paisaje, que al mismo tiempo hechiza y parece verdadero, tendría su correlato en la forma de expresión, no menos problemática, de ser a la vez poética y crítica a la poesía, y épica y a la vez crítica a la epopeya. Esto sería un 
rasgo caracterizador que Don Quijote compartiría con la novela moderna, como acentúa Octavio Paz, el cual adelanta con ello un pensamiento que encontraremos nuevamente y de forma más detallada en Carlos Fuentes (véase Simson 1989).

\section{Notas}

1 Fernández de Lizardi (1980: 14). Sobre la representación de la realidad social, véase Spell (1925: 145-165).

2 El apodo de "Quijotita", que en realidad se llama Pomposa, se lo habían puesto siete estudiantes amigos de su prima. Uno de ellos, apodado Sansón Carrasco, argumenta así: "Don Quijote era un loco y doña Pomposa es otra loca. Don Quijote tenía muy lúcidos intervalos en los que se explicaba bellamente, no tocándole sobre caballería; doña Pomposa tiene los suyos, en los que no desagrada su conversación; pero delira en tocándolo sobre puntos de amor y de hermosura" (Fernández de Lizardi 1973: 166/167).

3 Que las ideas avanzadas del Dr. Quix tienen que ser entendidas como una parodia de la extendida credibilidad en el progreso se pone de manifiesto cuando el Dr. Quix se refiere al ladrón que le ha robado a Sancho su nueva silla de montar en términos de "oxicéfalo" y "cloro-neurótico". Por esa razón no se le debe meter en la cárcel sino en un "Establecimiento de Antropología Penal". Véase Febres Cordero (1960: 146).

4 El título completo es "Civilización y barbarie: Vida de Juan Facundo Quiroga y aspecto físico, costumbres y hábitos de la República de Argentina", publicado primero en Obras publicadas bajo los auspicios del Gobierno Argentino, tomo VII, Buenos Aires 18851903.

5 Véase Rodó (1971); véase también el estudio introductorio de Leopoldo Alas (Clarín).

6 Asturias ve a Las Casas como la nueva personificación de Don Quijote. Las Casas también habría tenido que luchar como Don Quijote contra los molinos de la injusticia: "La locura de Don Quijote. Desfacer entuertos. Los dos Quijotes. El de Cervantes y el de Dios, en un solo Quijote. En ese gran sueño de justicia humana. Aquél perdió el seso leyendo libros de caballería, y éste, presenciando crímenes de caballeros. Su empeño es el mismo". (Asturias 1968: 7a). Ambos comparten la misma conciencia de misión y la misma intrepidez frente a sus enemigos. Su locura le parece a Asturias comparable a aquella que se le ha atribuido también a Jesucristo. Sería también característica de Bolívar, en el que Asturias ve un sucesor posterior de Las Casas.

7 Véase Zea (1952; 1955). Zea se basó en el estudiante del grupo de los "Ateneistas" Samuel Ramos, el cual en 1934 se planteó la cuestión de lo mexicano con El perfil del hombre y la cultura de México. Véase también Ripoll (1966: 5-29).

8 "Lo maravilloso, y con ello lo trascendente, surgió en el mundo de curas, bachilleres y barberos". (Zea 1988: 259).

9 Véase la reseña de Zea sobre la obra de Samuel Ramos Perfil del hombre y la cultura en México en Zea (1959: 24-31). 


\section{Bibliografía}

Alberdi, Juan Bautista. 1916. Peregrinación de Luz del Día o Viaje y aventuras de la Verdad en el Nuevo Mundo. Buenos Aires: La Cultura Argentina.

Asturias, Miguel Angel. 1968. 'Los dos Quijotes: la locura de Fray Bartolomé'. En: Excelsior (México, D.F.) (18 07 1968).

Darío, Rubén. 1950. 'Hércules y Don Quijote'. En: M. Sanmiguel Raimúndez (ed.), Obras completas I "Crítica y ensayo". Madrid: Afrodisio Aguado: 555-559.

- 1965. 'A Roosevelt'. En: Antonio Oliver Belmás (ed.), Azul...: el salmo de la pluma; cantos de vida y esperanza; otros poemas. México, D.F.: Porrúa: 123.

- 1970. 'D.Q.'. En: Roberto Ibáñez (ed.), Páginas desconocidas de Rubén Dario. Montevideo: Biblioteca de Marcha: 145.

Febres Cordero, Tulio. 1960. Don Quijote en América o sea la cuarta salida del ingenioso hidalgo de la Mancha. Bogotá: Antares.

Fernández de Lizardi, José Joaquín. ${ }^{2}$ 1973. La Quijotita y su prima. México, D.F.: Porrúa.

- 1980. El Periquillo Sarniento. Barcelona: Ramón Sopena.

Montalvo, Juan. 1972. Capitulos que se le olvidaron a Cervantes. Ensayo de imitación de un libro inimitable. México, D.F.: Porrúa.

Palau de Nemes, Graciela. 1981. 'DD.Q.': un cuento fantástico de Rubén Darío'. En: Manuel Criado de Val (ed.), Cervantes. Su obra y su mundo. Actas del I Congreso Internacional sobre Cervantes. Madrid: EDI-6: 943-947.

Paz, Octavio. 1973. El signo y el garabato. México, D.F.: Mortiz.

- 1988. 'Ambigüedad de la novela'. En: Frederick Viña (ed.), Don Quijote. Meditaciones hispanoamericanas I. Lanham, New York y London: University Press of America: 140-142.

Ripoll, Carlos. 1966. 'Samuel Ramos y la nueva imagen del hombre y de la cultura en México'. En: Cuadernos hispanoamericanos 68, 202: 5-29.

Rodó, José Enrique. 1950. 'El centenario de Cervantes'. En: Emir Rodríguez Monegal (ed.), Obras completas. Madrid: Aguilar: 1146-1148.

- $\quad{ }^{4} 1971$. Ariel. Madrid: Espasa-Calpe.

Simson, Ingrid. 1989. Realidad y Ficción en Terra Nostra de Carlos Fuentes. Madrid: Iberoamericana y Frankfurt am Main: Vervuert.

Spell, Jefferseon Rea. 1925. 'Mexican Society as seen by Fernández de Lizardi'. En: Hispania 8, 3: 145-165.

Uribe Echevarría, Juan. 1949. Cervantes en las letras hispano-americanas: antología y crítica. Santiago de Chile: Universidad de Chile.

Vasconcelos, José. 1947. 'Cervantes y América'. En: Revista iberoamericana 13: 1321.

Zea, Leopoldo. 1952. Conciencia y posibilidad del mexicano. México, D.F.: Porrúa. 1955. La filosofia en México. México, D.F.: Libro-Mex.

- 1959. 'Reseña'. En: Revista Nacional de Cultura (Caracas) 22: 24-31.

- 1988. 'Don Quijote y su lección sobre la realidad'. En: Frederick Viña (ed.), Don Quijote. Meditaciones hispanoamericanas I. Lanham, New York y London: University Press of America: 259-263. 